\title{
Review: Ginkgo biloba extract improves cognitive function in mild to moderate Alzheimer's disease
}

\author{
Oken BS, Storzbach DM, Kaye JA. The efficacy of Ginkgo biloba on cognitive function in Alzheimer disease. Arch Neurol 1998 \\ Nov;55:1409-15.
}

\section{Question}

In patients with Alzheimer's disease (AD), is Ginkgo biloba extract effective for improving cognitive function?

\section{Data sources}

Studies in all languages were identified by searching Medline, Embase/Excerpta Medica, and PsycLIT using the search terms ginkgo, gingko [sic], Tanakan, Tebonin, Rokan, Ginkoba, and EGb 761. Reference lists of relevant studies, textbooks, and a Cochrane Collaboration review were also used.

\section{Study selection}

Studies were selected if AD was diagnosed by using explicit criteria; exclusion criteria were clearly stated; standardised ginkgo extract ( $24 \%$ or $25 \%$ ginkgo-flavone glycosides and $6 \%$ terpenoids) in any stated dose and route of administration was used for $\geqslant 2$ months; the study was randomised, double blind, and placebo controlled; and $\geqslant 1$ outcome measure was an objective assessment of cognitive function. Studies were excluded if they did not state exclusion criteria for depression, other neurological disease, or medication that acted on the central nervous system.

\section{Data extraction}

Data were extracted on patient diagnoses, number of patients analysed, study duration, dropout rate, G biloba extract daily dose and formulation, cognitive outcome measures, other outcome measures, and results.

\section{Main results}

5 studies met the inclusion criteria, and 4 studies provided analysable data for 424 patients with mild to moderate $\mathrm{AD}$ (range
40 to 207 patients/study). Study duration was 12 weeks in 2 studies, 24 weeks in 1 study, and 26 weeks in 1 study (mean 19 wks). $G$ biloba doses were $120 \mathrm{mg}$ /day in 2 studies and 240 $\mathrm{mg} /$ day in 2 studies. 2 studies used the Synrom-Kurztest (SKT), 1 study used choice reaction time in addition to the SKT, 1 study used the Alzheimer Disease Assessment Scale-Cognitive subtest, and 1 study used a 10 item battery (which included the Benton Visual Retention Test, Digit Symbol, word list recall, and reaction time) to measure cognitive function. The pooled result showed that $G$ biloba extract was better than placebo for improving cognitive function $(\mathrm{p}<0.001)$ (table). No substantial adverse events occurred in patients in the $G$ biloba extract group.

\section{Conclusion}

In patients with mild to moderate Alzheimer's disease, Ginkgo biloba extract improves cognitive function.

Ginkgo biloba extract v placebo in mild to moderate Alzheimer's disease

\begin{tabular}{lll}
\hline $\begin{array}{l}\text { Outcome at mean follow } \\
\text { up of 19 weeks }\end{array}$ & Weighted mean difference & $\begin{array}{l}\text { Controls with a worse } \\
\text { outcome than treated }\end{array}$ \\
\hline Cognitive function & $0.41(0.22$ to 0.61$)$ & $66 \%(59$ to 73$)$ \\
\hline
\end{tabular}

*The proportion of control patients with a worse outcome than the average treated patient; calculated from data in article.

Source of funding: in part, National Institutes of Health.

For correspondence:Dr B S Oken,Department of Neurology, CR120, Oregon Health Sciences University, 3181 SW Sam Jackson Park Road, Portland, OR 97201, USA. Fax +1 5034844665.

\section{Commentary}

Extracts from the $G$ biloba tree have been used for 5000 years in traditional Chinese medicine to treat pulmonary diseases such as asthma and bronchitis. Curiously, the tree was the only plant to survive the bombing of Hiroshima and Nagasaki. ${ }^{1}$ Many French and German studies have reported on the efficacy of $G$ biloba for treating cerebral insufficiency or neurasthenia; an array of symptoms including memory impairment, depressed mood, confusion, light headed sensation, fatigue, tinnitus, and dizziness; and lack of motivation.

The positive effect of $G$ biloba on cognitive function in this review by Oken et $a l$ is not fully understood. The authors stressed that the 2 chemical components (flavonoid and terpenoid) of $G$ biloba have, respectively, an antioxidant profile and an anti-platelet activating factor (PAF) activity. Both mechanisms could be involved in therapeutic effects in AD. It has been claimed that free radicals are responsible for oxidative and peroxidase neuronal damage as well as for the amyloid deposition in $\mathrm{AD}$, which may be counteracted by $G$ biloba. The anti-PAF effect could counteract the proinflammatory effects and the extension of any vascular lesion derived by platelet activation, which are possible mechanisms in $\mathrm{AD}$. If these are the underlying mechanisms involved in the beneficial effect of $G$ biloba, the action should be limited to the early phases of the disease. Once the anatomical and morphological lesions are established with amyloid and neurofibrillary tangle deposition, no substance can stop the progression of symptoms. The authors did not report on the direct vascular effect of $G$ biloba on the arteries and small vessels, which is the reason it is prescribed for intermittent claudication. ${ }^{2}$ This may also explain the favourable effect of $G$ biloba in $\mathrm{AD}$ if, as some believe, there is an overlapping vascular component in this otherwise primary dementia.

Of note, the benefit of $G$ biloba in $\mathrm{AD}$ in this review is only slightly inferior to that shown in randomised controlled trials for acetylcholinesterase inhibitors, donepezil, ${ }^{3}$ and rivastigmine. ${ }^{4}$ These drugs have a totally different mechanism of action because they increase one of the neuromediators.

The authors of the primary studies are to be commended for using rigorous evaluation based on randomised controlled trials to understand the efficacy of this old remedy. Future studies on drugs in $\mathrm{AD}$ should measure more relevant outcomes, such as functional ones, to better evaluate their efficacy. Meanwhile, G biloba is a possible new approach in $\mathrm{AD}$.

Roberto Bernabei, MD Università Cattolica del Sacro Cuore Rome, Italy

1 Halpern GM. Ginkgo: a practical guide. Garden City Park, NY: Avery Publishing Group, 1998. 2 Peters H, Kieser M, Holscher U. Demonstration of the efficacy of Gingko biloba special extract EGB 761 on intermittent claudication-a placebo-controlled, double-blind multicenter trial. Vasa 1998;27:106-10.

3 Le Bars PL, Katz MM, Berman N, et al. A placebo-controlled, double-blind, randomised trial of an extract of Gingko biloba for dementia. JAMA 1997;278:1327-32.

4 Rosler M, Anand R, Cicin-Sain A, et al. Efficacy and safety of rivastigmine on patients with and safety, of rivastigmine on patients with controlled trial. BMJ 1999;318:633-8. 